EVALUATION OF SERUM ALKALINE PHOSPHATASE (ALP) AND CALCIUM LEVEL IN RHEUMATOID ARTHRITIS- A COMPARATIVE STUDY
KEY WORDS: Rheumatoid arthritis, Calcium, Alkaline phosphatase.
Aishwarya KP*

\section{Reshmy GS}

Department of Biochemistry, Amrita Institute of Medical sciences and Research Centre, Amrita vishwa vidyapeetham, Kochi, Kerala, India. *Corresponding Author

Department of Biochemistry, Amrita Institute of Medical sciences and Research Centre, Amrita vishwa vidyapeetham, Kochi, Kerala, India.

Background: Rheumatoid arthritis is an autoimmune disease that can cause joint pain and damage throughout the body. Rheumatoid arthritis affects about $1 \%$ of world population. There will be variation in calcium and alkaline phosphatase levels in rheumatoid arthritis patients. So estimation of these two parameters helps in the diagnosis of the severity of rheumatoid arthritis.

Aim \& Objective: The aim of the study is to find the levels of calcium and alkaline phosphatase in rheumatoid arthritis patients and comparing it with healthy controls to find any deviation from its normal level.

Materials \& Methods: A total of 30 clinically established rheumatoid arthritis patients and 30 healthy age matched control were involved in the study. Serum of the patients and controls were assayed for alkaline phosphatase levels and calcium levels.

Result: This study shows an elevated alkaline phosphatase levels and a diminished calcium levels in patients with rheumatoid arthritis when compared to the control.

Conclusion: Since the study shows a significant variation in alkaline phosphatase and calcium levels it can serve as a less costly marker for assessing the severity of rheumatoid arthritis.

\section{INTRODUCTION}

Rheumatoid arthritis is a chronic inflammatory autoimmune disease with progressive course in which arthritis results from own immune system and attacking mainly synovium (the lining of the membrane that surrounds joints) and it affects $1 \%$ of the world's adults. RA affects the articular and extra articular structures resulting in pain, disability and mortality. The immune system usually synthesis antibodies against microbes like bacteria and viruses. But in case of rheumatoid arthritis, immune system mistakenly sends antibodies to the lining of joints where they attack the tissue surrounding the joint. This causes the synovium to become sore and inflamed, releasing chemicals that damage bones, cartilage, tendons and ligaments. Persistent inflammation leads to erosive joint damage and functional impairment in vast majority of patients ${ }^{[1]}$. If rheumatoid arthritis is not treated, these chemicals gradually cause the joint to lose its shape and alignment and eventually can destroy the joint completely.

Alkaline phosphatase is an enzyme with multisystem existence as its various isoenzymes are found in different tissues of the body. Half of the total alkaline phosphatase in serum is derived from bone. In RA patients there is an increased ALP level, especially in women. It may be due to degenerative changes in the wrist joint and other small joints leads to autoimmune erosive changes in bone. A compensatory mechanism in turn comes into action to restore the destroyed bone in the joints leading to proliferation of osteoblasts and hence the elevated ALP activity. A sub stained part of RA patients have hypocalcaemia. Hypocalcaemia is associated with high disease activity and may contribute to suppression of parathyroid hormone secretion and vitamin $\mathrm{D}$ hormone synthesis. High levels of ionized calcium may be a reflection of disease activity related systemic bone loss and could be a predictor of decreased bone mineral density at the lumbar spine in RA.

In our study, therefore, proposed to observe, two cardinal markers of bone formation, namely alkaline phosphatase and calcium levels in serum of patients with rheumatoid arthritis for any deviations from their normal levels.

\section{MATERIAL}

The study was carried out at Amrita Institute of Medical Sciences, Cochin. This was a retrospective laboratory based study. The study period was for 6 months from October 2019 to March 2020. Data for the study was collected from the clinical laboratory attached to the hospital.

A total of 30 samples from clinically diagnosed RA patients (based on their RF value, Anti CCP value and physician's progress note) over a period of 6 months between ages of 20 50 years from both gender were included in the study. 30 healthy age matched controls were also included.

Patients with chronic renal failure, malignancy, diabetes mellitus, HIV, HCV, pregnant women, age below 20 and above 50 were excluded.

\section{METHOD}

Colorimetric assay in accordance with a standardized method was used for both ALP and Calcium estimation.

\section{RESULT}

Statistical Analysis

Statistical analysis was done using IBM SPSS 20 (SPSS Inc, Chicago, USA). For all the continuous variables, the results are either given in Mean \pm SD and for categorical variables as percentage. To obtain the association of categorical variables, chi square test was applied. To compare the mean difference of numerical variables between groups, independent two sample't' test was applied. A P-value $<0.05$ was considered as statistically significant.

Table: 1 Comparison Of Mean Calcium Levels (mg/dl) Between Groups

\begin{tabular}{|l|l|l|l|}
\hline GROUP & TOTAL NUMBER & MEAN+/-SD & P-VALUE \\
\hline CASE & 30 & $8.43 \pm 0.679$ & 0.002 \\
\cline { 1 - 2 } CONTROL & 30 & $9.00 \pm 0.695$ & \\
\cline { 1 - 2 } & &
\end{tabular}

Table: 2 Comparison Of Mean Alp Levels (IU/L) Between Groups

\begin{tabular}{|l|l|l|l|}
\hline GROUP & TOTAL NUMBER & MEAN SD & P VALUE \\
\hline CASE & 30 & $93 \pm 27.127$ & $<0.001$ \\
\hline CONTROL & 30 & $62 \pm 16.293$ & \\
\hline
\end{tabular}

Table: 3 Comparison Of Gender Distribution (\%) In Case.

\begin{tabular}{|l|l}
\hline GENDER & PERCENTAGE (\%)
\end{tabular}

\begin{tabular}{|l|l|}
\hline FEMALE & $60 \%$ \\
\hline
\end{tabular}

\begin{tabular}{|l|l|}
\hline MALE & $40 \%$ \\
\hline
\end{tabular}




\section{DISCUSSION}

This retrospective study involves 30 clinically diagnosed rheumatoid arthritis patients on the basis of them being tested positive for rheumatoid factor (RF), serum Anti CCP level and based on a physician diagnosis progress note and 30 healthy age matched control. The calcium and ALP levels were measured in both case and control.

The study shows (Table: 1 ) a significant $(P=0.002)$ decrease in serum calcium level in rheumatoid arthritis patients as compared to the age matched control. This may be due to an increased rate of calcium metabolism, increased excretion of calcium through urine in RA patients and impaired calcium absorption related to intestinal defect. The primary reduction in calcium absorption increases the risk of osteoporosis. Also circulating pro inflammatory molecules \& hormones that alter calcium metabolism contribute to the diminished calcium status.RA is usually associated with generalized bone mineral density (BMD) loss, erosions and localized or generalized osteoporosis, resulting in increased risk of clinical fractures and functional disability. These results indicate that high disease activity in patients with rheumatoid arthritis is associated with an alteration in calcium levels and increased bone resorption.

The level of ALP (Table: 2 Figure: 2 ) in RA patients showed a significant $(P<0.001)$ increase in RA patients as compared to the healthy age matched control. This elevation of alkaline phosphatase in rheumatoid arthritis patients is probably due to degenerative changes in the wrist joint \& other small joints leading to auto-immune erosive changes in bone. Elevated ALP in RA has been attributed to osteoblastic activity indicating an increased bone turnover. Also the promotion of disease activity is known to induce more active bone resorption, which is accompanied by a rise in serum ALP and concomitant bone formation. Increased activity of ALP may be due to its leakage from injured or killed cells. As a compensatory mechanism for the changes in bone, body start to restore the destroyed bone in the joints, leading to proliferation of osteoblasts \& hence the elevated alkaline phosphatase activity. Thus rheumatoid arthritis can provide an ideal platform for osteoporosis to sets in as the already eroded bone becomes brittle as a consequence of the autoimmune destruction making the patient more susceptible to fractures.

In this present study of $30 \mathrm{RA}$ patients (Table No: 3 ) $60 \%$ were females and $40 \%$ were males.The reasons for this over representation of women are not clear, but genetic (X-linked) factors and hormonal aspects are likely to be involved. These gender differences in RA may be firstly because women get autoimmune diseases in far greater number than men as the female immune system is stronger and more reactive.

\section{CONCLUSION}

This study reveals a markedly elevated alkaline phosphatase and a decreased calcium levels in rheumatoid arthritis patients as compared to the control. These biochemical alterations reflect on the pathogenesis of RA. The increase in alkaline phosphatase is explained by degenerative changes in wrist joint and other small joints. The decrease in calcium level may be due to the increased rate of bone metabolism and excretion of calcium through urine. Thus, understanding the pathophysiology of rheumatoid arthritis may help to develop a new therapeutic approach in the management of the disease. Therefore, serum ALP \& calcium can be used as markers in assessing severity of rheumatoid arthritis.

\section{REFERENCE}

1) Behzad Heidari, Rheumatoid Arthritis: Early diagnosis and treatment outcomes.Caspian J Intern Med. 2011 winter;2(1): 161-170.

2) Harris ED Jr. Pathogenesis of rheumatoid arthritis.Am J Med. 1986 Apr 28; 80(4B):4-10.

3) David A Fox, Alison Gizinski, Rachel Morgan, Steve K Lundy. Cell-cell interactions in rheumatoid arthritis synovium. Rheum Dis Clin North Am. 2010 May;36(2):311323.

4) Anirban Poddar, Debasis Debadatta Behera, Sudeshna Ray. Serum alkaline phosphatase activity and serum calcium level: an assessment tools for disease activity in rheumatoid arthritis. Indian Journal of Basic and Applied Medical Research September 2016:Vol.-5, Issue-4, P. 132-134.

5) M.J.Kendall, R.Cockel, J.Becker and C.F.Hawkins. Raised serum alkaline phosphatase in rheumatoid arthritis, an index of liver function.Ann. rheum. Dis. (1970), 29, 537

6) D.L.Scott, M.Farr, C.F.Hawkins, R.Wilkinson, A.M.Bold. Serum calcium levels in rheumatoid arthritis; from the rheumatism research wing and departments of medicine and clinical chemistry. Annals of the Rheumatic Diseases, 1981, 40,580-583.

7) Niino-Nanke Y, Akama H, Hara M, Kashiwazaki S. Alkaline phosphatase activity in rheumatoid arthritis; its clinical significance and synthesis of ALP in RA Synovium.Ryumachi. 1998 Aug; 38(4):581-8.

8) Sumihisa Aida. Alkaline phosphatase isoenzyme activities in rheumatoid arthritis: hepatobiliary enzyme dissociation and relation to disease activity. Ann Rheum Dis. 1993 Jul; 52(7):51 1-516.

9) Perlik F, Kutova M. The evaluation of bone changes in patients with rheumatoid arthritis.Z Rheumatol. 1983 Sep-Oct; 42(5):261-4.

10) Marian W Ropes, Elsie C.Rossmeisl and Walter Bauer. Calcium and phosphorous metabolism in rheumatoid arthritis and degenerative joint disease.J Clin Invest. 1943 Nov; 22 (6):785-790.

11) P N Sambrook, G Abayasekara, B M Ansell, S Foster, J M Gumpel, P A Hill, J Reeve, and J C Stevenson. Calcium absorption in rheumatoid arthritis. Annals of the Rheumatic Diseases 1985,44,585-588.

12) Vaithialingam A, MohanaLakshmi T, Suryaprakash G, Edukondalu AD, Prabhakar Reddy E. Alkaline phosphatase levels in rheumatoid arthritis \& osteoporosis in clinical practice. Journal of current trends in clinical medicine \& Laboratory Biochemistry, 2013; 1, (2):20-23.

13) M G Bramble, D R Blanke, T White, J Sly, D N S Kerr. Ionized calcium in rheumatoid arthritis: effect of non steroidal anti inflammatory drugs. Br Med J. 1980 Sep 27;281(6244):840-841.

14) NankeY, Kotake S, Akama H, Kamatani N. Alkaline Phosphatase in Rheumatoid Arthritis Patients: Possible Contribution of Bone-Type ALP to the Raised Activities of ALP in Rheumatoid Arthritis Patients. Clin Rheumatol. 2002 Jun; 21(3):198-202.

15) Beyeler C, Banks RE, Thompson D, Forbes MA, Cooper EH, Bird H. Bone alkaline phosphatase in rheumatic diseases. Ann Clin Biochem. 1995 Jul; 32 (Pt 4):379-84. 